\title{
Evaluating speech samples designed for the Voice Profile Analysis Scheme for Brazilian Portuguese (BP-VPAS)
}

\author{
Zuleica Antonia de Camargo ${ }^{1}$, Sandra Madureira1, Luiz Carlos Rusilo ${ }^{2}$ \\ ${ }^{1}$ Integrated Acoustic Analysis and Cognition Laboratory-LIAAC-Pontifical Catholic \\ University of São Paulo, Brazil \\ ${ }^{2}$ Department of Actuarial and Quantitative Methods-DAMQ-Pontifical Catholic \\ University of São Paulo, Brazil \\ https://doi.org/10.36505/ExLing-2011/04/0012/000181
}

\begin{abstract}
The present study aims at evaluating a corpus designed for voice quality settings analysis (Laver, 1980). It addresses the role of keyspeech segments for the identification of voice quality settings. The corpus, recorded by 60 speakers, contained repetitions of 10 keyspeech sentences, and semi-spontaneous speech samples. These samples were evaluated by two expert subjects according to the BP-VPAS Profile (Camargo, Madureira, 2008). These data were analyzed by means of an artificial neural network and statistically by multiple regression analysis. The data reinforce the importance of the description of voice quality based on the phonetic model and the relevant role of keyspeech segments.
\end{abstract}

Key words: voice quality; auditory perception, phonetics, evaluation; corpus

\section{Introduction}

This study aims at evaluating a corpus designed for voice quality evaluation, based on the phonetic description of voice quality model (Laver, 1980), addressing the role of keyspeech segments in detecting voice quality settings in Brazilian Portuguese context. The Vocal Profile Analysis Scheme- VPAS, which is based on a phonetically description of voice quality (Laver, 1980), is the result of the continuous work of a team of researchers from Queen Margareth University CollegeEdinburgh (Laver et al, 1981; Laver, 2000; Laver, Mackenzie-Beck, 2007).

The application of the VPAS at the Brazilian context to investigating linguistic, paralinguistic and extralinguistic uses of vocal quality and acoustic and physiological correlates of settings of voice quality besides the experience derived from structuring a voice quality database, from introducing the model in workshops and from answering questions, posed by learners in these workshops, about voice data collection procedures and application procedures, led a group of researchers at LIAAC- PUCSP (Integrated Acoustic Analysis and Cognition Laboratory of the Catholic University of São Paulo) to systematize and improve the material aimed at instructional purposes (Camargo, Madureira, 2008).

The phonetic approach to voice quality, based on the susceptibility of speech segments to the effects of voice quality settings was considered in order to elaborate a corpus of 10 keyspeech sentences (Camargo,

ExLing 2011: Proceedings of 4th Tutorial and Research Workshop on Experimental Linguistics, 25-27 May, Paris, France 
Madureira, 2008) specially designed for auditory voice quality evaluation by VPAS, as an adaptation for Brazilian context (BP-VPAS).

This adaptation of the VPAS to the Brazilian Portuguese context followed a comprehensive theoretical critical review of the bases of the model profile. The adaptation of the terminology took into account advances in speech science research related to studies on the physiology and speech signal research (Hammarberg, Gauffin, 1995; Chasaide, Gobl, 2005).

\section{Methods}

The corpus was recorded by 60 speakers and retrieved in the voice database, being 12 men and 48 women averaged 39,7 years-old. (19 to 58). It contained repetitions of 10 keyspeech sentences (ks) built with keyspeech segments (named as ks fala, ks cidadeSP, ks Roberta, ks Lara, ks Liliane, ks garoto, ks Cbispos, ks detest, ks Santos, and ks filosofia) and semispontaneous speech samples as answers to the request: "Talk about the city where you were born". These samples were recorded in a radio studio and analysed by means of perceptual (vocal profiles in terms of vocal tract, tension and phonatory settings) and acoustic (f0 measures- median, quartil semi-amplitude and $99,5 \%$ quantil- extracted automatically by means of Expression Evaluator script- Barbosa, 2009- version 2011) procedures.

These data were analyzed by means of an artificial neural network and statistically by multiple regression analysis, in order to investigate the speech samples (keyspeech sentences) as related to the evaluation of voice quality settings (in scalar degrees between 1 and 6).

\section{Results}

The results contemplate some correlations, detected by means of statistical analysis, between perceived voice quality settings and characteristics of the keyspeech sentences of the corpus. The degree of voice quality settings was found to be related to their incidence in the distinct sentences evaluated.

Almost all the keyspeech sentences were suitable for analyzing both phonatory and non-phonatory voice quality settings evaluated with degree 3 or higher (Figure 1): tongue tip/blade (advanced); jaw (minimized range), tension (laryngeal hyperfunction) and phonatory (creaky and harsh voice).

For the purpose of differentiating degrees lower than 3, the keyspeech sentences were useful to detect distinct lip (spreading, labiodentalization and minimized range), jaw (open), tongue tip/blade (retracted), tongue body (fronted, backed, lowered and minimized range), velopharyngeal (nasal), pharynx-constriction/expansion and tension (vocal tract hyperfunction) voice quality settings. 
The semi-spontaneous speech samples allowed the identification of lip spreading, and labiodentalization, advanced and lowered tongue body, nasal, lowered larynx, and breathy voice quality settings (Figure 1).

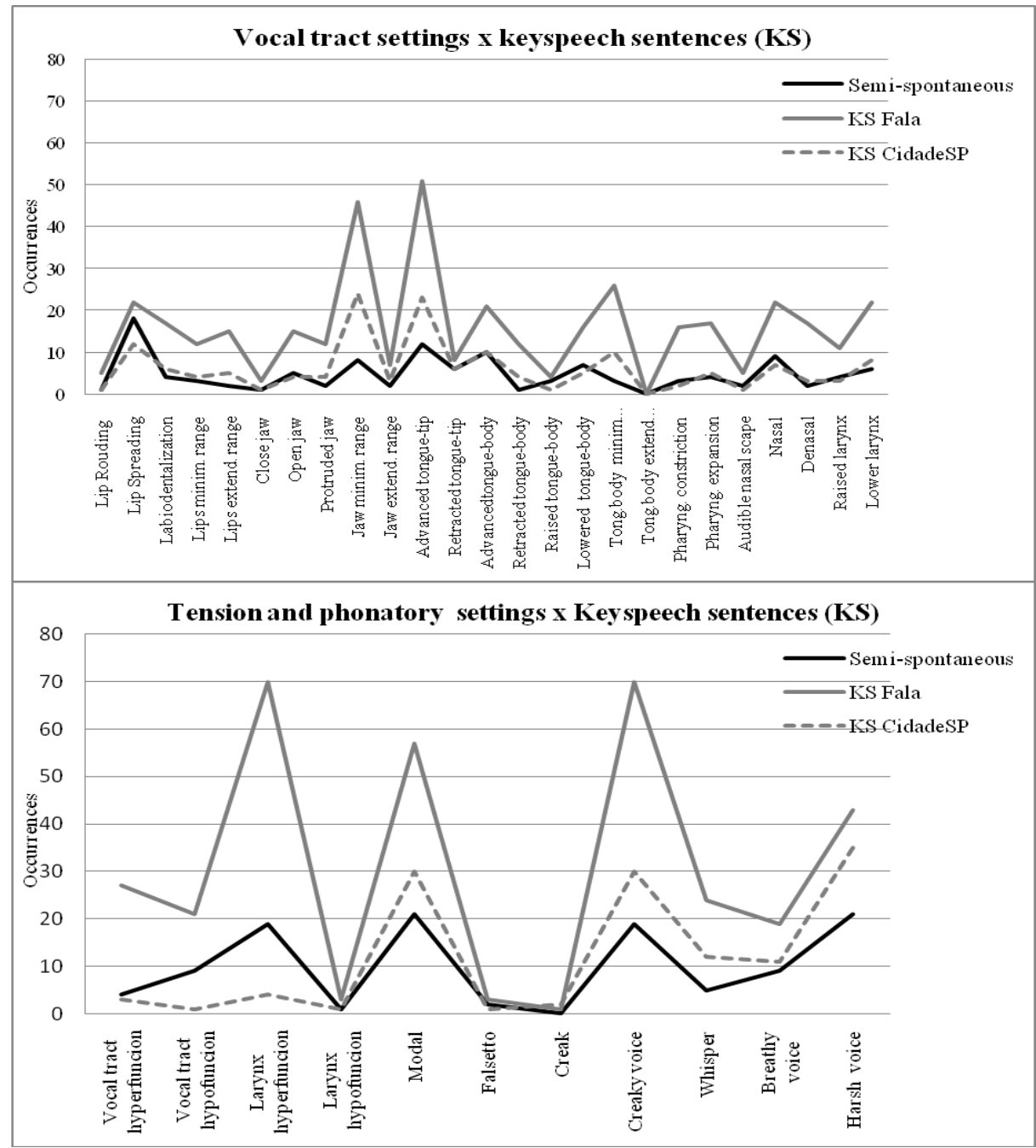

Figure 1. Perceptual evaluation of vocal tract, tension and phonatory settings based on keyspeech sentences (ks) and semi-spontaneous corpus.

For the group of $\mathrm{f0}$ measures, it was found that median values of $\mathrm{f} 0$ of $\mathrm{ks}$ fala were related to that found in semi-spontaneous recordings. However, the f0 values of all the other keyspeech sentences turn out to be higher. The quartile amplitude were similar between semi-spontaneous samples and $k s$ 
detesto, ks santos and ks filosofia. The semi-spontaneous samples and $k s$ Roberta were found to be similar in relation to the $99,5 \%$ quantil.

The ks fala ( $O$ objeto de estudos da fonética é esta complexa, variável e poderosa face sonora da linguagem: a fala) allowed the identification of a wide range of voice quality settings, probably due to its composition. It contained as key segments all oral vowel sounds, three nasal vowels, fricatives, plosives, liquids and nasals). Furthermore it presented no difficulties to be produced.

\section{Conclusions}

The data reinforce the importance of the description of voice quality based on the phonetic model and the relevant role of some keyspeech segments in the corpus designed for voice quality evaluation.

\section{Acknowledgements}

We acknowledge Plínio Barbosa from UNICAMP for SG Expression Evaluator Script- 2011 Version to the database. This study has been supported by Research and Teaching Comission grant at Pontifical Catholic University of São Paulo (CEPE-PUCSP: 09/639).

\section{References}

Barbosa, P. A. 2009. Detecting changes in speech expressiveness in participants of a radio program In Proc. of Interspeech. v.1,2155-2158. Brighton, Reino Unido.

Camargo, Z., Madureira, S. 2008. Voice quality analysis from a phonetic perspective: Voice Profile Analysis Scheme Profile for Brazilian Portuguese (BP VPAS). In Proc.of the $4^{\text {th }}$ Conf on Speech Prosody v.1, 57-60. Campinas, Brazil.

Chasaide,N.A.; Gobl, C. 2005. On the relation between phonatory quality and affect. In Hardcastle, W.J., Mackenzie-Beck, J.(eds). A figure of speech: a festschrift for John Laver. Mahwah: Lawrence Erlbrum, 323-346.

Hammarberg, B., Gauffin, J. 1995. Perceptual and acoustics characteristics of quality differences in pathological voices as related to physiological aspects. In Fujimura, O. and Hirano, M (eds), 1995, Vocal fold physiology, 283-303. San Diego, Singular Publishing.

Laver, J. 1980. The phonetic description of voice quality. Cambridge University Press. Reprinted.2009.

Laver, J. 2000. Phonetic evaluation of voice quality. In: Kent, R.D., Ball, M.J. (eds) The handbook of voice quality measurement. San Diego, CA: Singular publications.

Laver, J., Wirs, S., Mackenzie, J., Hiller, S.M. 1981. A perceptual protocol for the analysis of vocal profiles. Edinburg University Department of Linguistics Work in Progress 14,139-155.

Laver, J; Mackenzie-Beck J. 2007. Vocal Profile Analysis Scheme -VPAS. Queen Margareth University College-QMUC, Speech Science Research Centre, Edinburgh. 